\title{
Robotic resection of a middle mediastinal mass
}

\author{
Marco Nardini ${ }^{1}$, Joel Dunning ${ }^{2}$, Marcello Migliore ${ }^{1}$, Robert J. Cerfolio ${ }^{3}$ \\ ${ }^{1}$ Department of Surgery, Division of Thoracic Surgery, University Hospital of Catania, Catania, Italy; ${ }^{2}$ Department of Cardiothoracic Surgery, James \\ Cook University Hospital, Middlesbrough, UK; ${ }^{3}$ Department of Cardiothoracic Surgery, New York University Langone Health, New York, USA \\ Correspondence to: Marco Nardini. Department of Surgery, Division of Thoracic Surgery, University Hospital of Catania, Catania, Italy. \\ Email: marco.n@doctors.org.uk.
}

\begin{abstract}
Aorto-pulmonary paraganglioma is an exceptionally rare condition, and its diagnosis and treatment are a challenge for the general thoracic surgeon. We describe the case of a 35 years old man who was incidentally diagnosed with a visceral mediastinal mass, deeply encased in the aorto-pulmonary window. To our knowledge this is the first case of its kind to be successfully treated with the adoption of a minimally invasive technique. We conclude that the dissection was made easier by the robotic instrumentation and by the camera system, and a minimally invasive approach would have been more difficult by traditional thoracoscopy.
\end{abstract}

Keywords: Robotic thoracic surgery; robotic surgery; minimally invasive thoracic surgery (MITS); mediastinal mass; neuroendocrine tumors

Received: 24 April 2018; Accepted: 02 May 2018; Published: 25 May 2018.

doi: 10.21037 /jovs.2018.05.13

View this article at: http://dx.doi.org/10.21037/jovs.2018.05.13

\section{Introduction}

Tumors that origin from the aorto-pulmonary paraganglia are extremely rare, and the overall incidence represent less than $1 \%$ of all the mediastinal neoplasms (1). These neoplasms belong to the group of neuroendocrine tumors. They are divided in functional and non-functional paragangliomas on the basis of catecholamines production and secretion. Pheochromocytoma is the most frequent paraganglioma, which arises from the chromaffin cells of the adrenal medulla, and, unlike its mediastinal counterpart, often produces catecholamines and cause a precise clinical syndrome. Functional mediastinal paragangliomas are rarer and usually arise in the posterior mediastinum (2). Aortopulmonary paragangliomas are diagnosed when the growth compresses the surrounding structures or as an incidental finding, like in our case. This masses have a rich (3) and sometimes aberrant (4) blood supply which make surgical resection challenging. Furthermore, if located in the aorta-pulmonary window, the resection is particularly difficult either by open or minimally invasive technique and occasionally cardiopulmonary by-pass has been used (5). To our knowledge this is the first case of aorto-pulmonary paraganglioma to be resected by minimally invasive approach, namely with the aid of the robotic platform.

\section{Case presentation}

We describe the case of a 35 -years-old man diagnosed with a $4.5 \mathrm{~cm}$, well defined, rounded mass in the visceral mediastinum. This was found by a chest computed tomography scan performed as part of a coronary screening (Figure 1). This patient had no other previous medical history of note. The mass was encased deeply in the aorto-pulmonary window as described in the video. It was surrounded by the arch of the aorta, sitting over the pulmonary trunk, anterior to the carina and posterior to the ascending aorta. Its superior aspect was lateral to the left side of the distal trachea. A biopsy obtained with endo-bronchial ultrasound (EBUS) was described by the pathologist as carcinoid tumor. This initial diagnosis was unlikely on the basis of the anatomical location. The patient was proposed removal by mean of median sternotomy in two other institutions, he was committed to have a minimally invasive procedure and so chased a third opinion. Professor Cerfolio gave indication for surgery, both for diagnostic and therapeutic intent, and proposed a left robotic approach at New York University, Langone Medical Centre. The patient accepted a risk of hoarseness of voice up to $50 \%$. Preoperative plasmatic level of metanephrines was normal. In the operating room the patient was positioned in lateral 


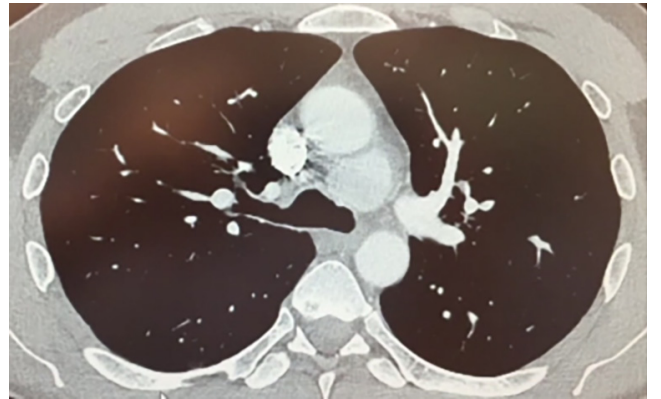

Figure 1 Chest computed tomography which shows the incidental mass in front of the carina, posterior to the ascending aorta and sitting over the pulmonary trunk's bifurcation.

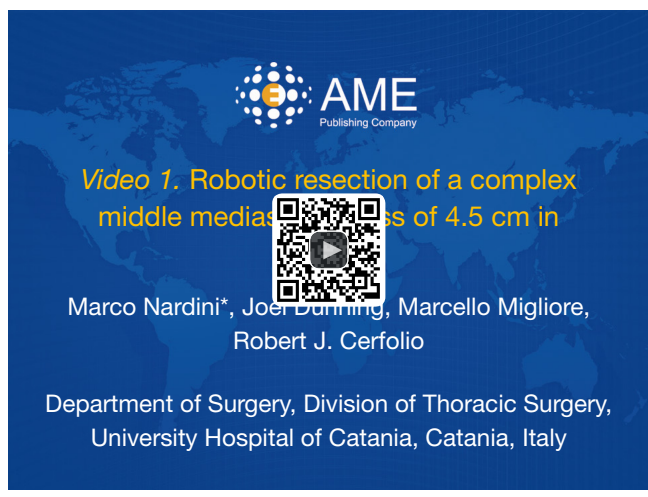

Figure 2 Robotic resection of a complex middle mediastinal mass of $4.5 \mathrm{~cm}$ in diameter (7). The robotic platform allowed a minimally invasive approach. The chest drain was removed on the following day, when the patient was discharged home.

Available online: http://www.asvide.com/article/view/24951

decubitus after selective intubation. The ports were placed similarly to what previously described (6) such as: all the robotic ports were placed over the eight rib with the posterior port performed $4 \mathrm{~cm}$ from the spinal process and connected to arm number $4,8 \mathrm{~cm}$ anteriorly is the port for arm number 3 , then, $8 \mathrm{~cm}$ away from the previous one, it was the camera port, and finally, as lower and as anterior as possible, it was the port for arm number 1 . The assistant's port was placed between the least two but one intercostal space below (Figure 2). The robot (Xi DaVinci System, Intuitive, CA, USA) was now ready to be docked over the patient. A bipolar dissector was placed in arm 3, a Cadiere forceps in arm 1, a curved up grasper in arm 4. A 0-degree camera was adopted as this reduce the torque on the rib.

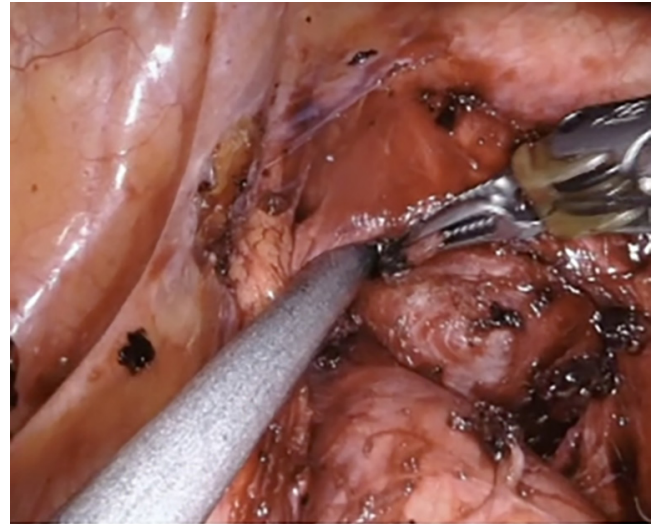

Figure 3 The lateral aspect of the mass is exposed in the aortopulmonary window.

The dissection started opening the mediastinal pleura over the left vagus nerve, which was promptly identified in its course. Lymph node region 5 is dissected. After further dissection medially, the smooth surface of the lateral aspect of the mass was finally identified (Figure 3). The curved up grasper it was used for retraction of the lung inferiorly, while the bed side assistant used the suction and passed tools, like cigar sponges and sutures, trough the assistant port. Once the mass was visualized the technical key, in order to find a plane of dissection, it has been to pass a suture through the neoplasm. This could allow retraction in all the direction and so perform the dissection between the capsule of the mass and the surrounding structures. The needle caused a small amount of bleeding from the capsule which obscured the field initially. After careful blunt and sharp dissection, performed mainly with the bipolar dissector and allowed by the retraction of the lesion with the Cadiere forceps holding the suture, the mass was then freed and ready to be placed in an endo-bag and delivered through the camera port (Figure 4). An apical $28 \mathrm{Fr}$ chest drain was placed through the assistant port soon after dedocking of the robot and prior to routine closure. The procedure was 120 minutes long and the blood loos was not clinically significant. The chest drain was ablated on the following day and the patient was discharged home. He complained of some degree of hoarseness which is gradually resolving without laryngology intervention. This was likely due to trauma to the left recurrent laryngeal nerve, which was not actually compromised by the resection. The final histology described a primary mediastinal paraganglioma. The patient is well 2 months after surgery. 


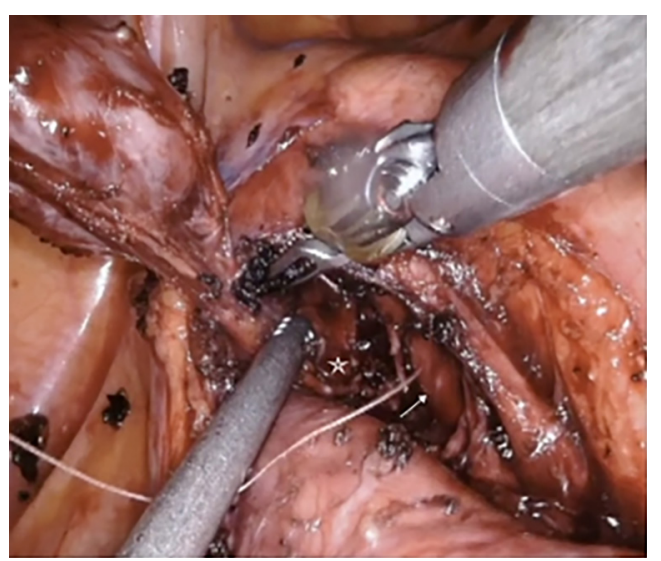

Figure 4 The mass is almost completely mobilized from the visceral mediastinum and it is retracted at the top left of the picture. The arrow points the esophagus and the star is over the trachea which was tightly adherent to the lesion.

\section{Comments}

Aorto-pulmonary paraganglioma is an extremely rare condition which diagnosis and management are a real challenge for the thoracic surgeon. Surgical resection is the accepted gold standard of treatment but can be technically very difficult. The capsule of the paraganglioma can have a rich vascularization and also aberrant vascular supply has been described. Mediastinal paraganglioma rarely secretes catecholamines but arterial blood pressure changes, during mobilization, should be considered. These aspects should be kept in mind when approaching these rare cases.

To our knowledge this is the first case of its kind to be performed through a port assess, minimally invasive approach. The robotic platform, in experienced hands, with articulated instruments and enhanced view allowed to perform safely the procedure while maintaining a minimally invasive approach. To perform the resection of a similar mass, deeply located in the visceral mediastinum, and tightly adherent to the vital surrounding structures, could have been more challenging if not impossible with rigid shaft traditional thoracoscopic instruments. The robot, on the other hand, can simplify the procedure, the structure is visualized better than in open surgery, the recovery is enhanced by reducing the surgical trauma to the patient, and the cosmetic outcome is obviously superior.

\section{Acknowledgements}

The authors would like to thank all the staff involved in the care of this patient at New York University Langone Health.

\section{Footnote}

Conflicts of Interest: Prof Cerfolio discloses a financial relationship with Ethicon, Covidien, Intuitive, C-SATS, Bovie, Community Health Services, Bard, Myriad Genetics, and Rolo-7 Company, LLC. The other authors have no conflicts of interest to declare.

Informed Consent: Written informed consent was obtained from patient for publication of this report and any associated images and videos.

\section{References}

1. Balcombe J, Torigian DA, Kim W, et al. Cross-sectional imaging of paragangliomas of the aortic body and other thoracic branchiomeric paraganglia. AJR Am J Roentgenol 2007;188:1054-8.

2. Ma L, Mei J, Liu L. Thoracoscopic resection of functional posterior mediastinal paraganglioma: a case report. J Thorac Dis 2014;6:1861-4.

3. Wald O, Shapira OM, Murar A, et al. Paraganglioma of the mediastinum: challenges in diagnosis and surgical management. J Cardiothorac Surg 2010;5:19.

4. Matsumoto J, Nakajima J, Takeuchi E, et al. Successful perioperative management of a middle mediastinal paraganglioma. J Thorac Cardiovasc Surg 2006;132:705-6.

5. Lamy AL, Fradet GJ, Luoma A, et al. Anterior and middle mediastinum paraganglioma: complete resection is the treatment of choice. Ann Thorac Surg 1994;57:249-52.

6. Cerfolio RJ, Watson C, Minnich DJ, et al. One Hundred Planned Robotic Segmentectomies: Early Results, Technical Details, and Preferred Port Placement. Ann Thorac Surg 2016;101:1089-95; Discussion 1095-6.

7. Nardini M, Dunning J, Migliore M, et al. Robotic resection of a complex middle mediastinal mass of $4.5 \mathrm{~cm}$ in diameter. Asvide 2018;5:520. Available online: http:// www.asvide.com/article/view/24951

doi: 10.21037/jovs.2018.05.13

Cite this article as: Nardini $M$, Dunning J, Migliore $M$, Cerfolio RJ. Robotic resection of a middle mediastinal mass. J Vis Surg 2018;4:113. 